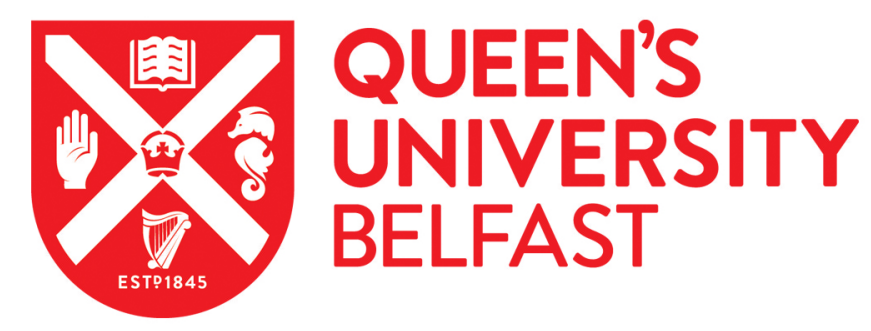

\title{
RBPF-MSIS: Towards Rao-Blackwellized Particle Filter SLAM for Autonomous Underwater Vehicle with Slow Mechanical Scanning Imaging Sonar
}

Chen, L., Yang, A., Hu, H., \& Naeem, W. (2020). RBPF-MSIS: Towards Rao-Blackwellized Particle Filter SLAM for Autonomous Underwater Vehicle with Slow Mechanical Scanning Imaging Sonar. IEEE Systems Journal, 14(3), 3301-3312. https://doi.org/10.1109/JSYST.2019.2938599

Published in:

IEEE Systems Journal

Document Version:

Peer reviewed version

Queen's University Belfast - Research Portal:

Link to publication record in Queen's University Belfast Research Portal

Publisher rights

Copyright 2019 IEEE. This work is made available online in accordance with the publisher's policies. Please refer to any applicable terms of use of the publisher.

\section{General rights}

Copyright for the publications made accessible via the Queen's University Belfast Research Portal is retained by the author(s) and / or other copyright owners and it is a condition of accessing these publications that users recognise and abide by the legal requirements associated with these rights.

Take down policy

The Research Portal is Queen's institutional repository that provides access to Queen's research output. Every effort has been made to ensure that content in the Research Portal does not infringe any person's rights, or applicable UK laws. If you discover content in the Research Portal that you believe breaches copyright or violates any law, please contact openaccess@qub.ac.uk. 


\title{
RBPF-MSIS: Towards Rao-Blackwellized Particle Filter SLAM for Autonomous Underwater Vehicle with Slow Mechanical Scanning Imaging Sonar
}

\author{
Ling Chen, Aolei Yang*, Huosheng Hu,Wasif Naeem
}

\begin{abstract}
Simultaneous Localization and Mapping (SLAM) has the potential to play a fundamental and significant role in achieving autonomy for Autonomous Underwater Vehicles (AUV). This paper proposes a Rao-Blackwellized Particle Filter (RBPF) SLAM algorithm for an AUV equipped with a Mechanically Scanning Imaging Sonar (MSIS) that has a very slow scanning frequency. To tackle the issues of scan distortion and sonar data sparseness caused by the slow-sampling MSIS, the core of the algorithm is a carefully designed sliding window based scan forming module. Then the formed scans are fed into the modified RBPF to build a consistent grid-based map thus localizing the AUV accurately. Extensive simulation and experiments are carried out to verify the proposed algorithm. The results show that the proposed algorithm outperforms existing ones in terms of the level of map consistency with the environment as well as the accuracy of pose estimation.
\end{abstract}

Index Terms-Autonomous Underwater Vehicle, SLAM, Mechanical Scanning Image Sonar, Rao-Blackwellized Particle Filter.

\section{INTRODUCTION}

A Utonomous Underwater Vehicles (AUVs) have been applied for searching underwater resources and conducting various tasks such as underwater rescue [1], map building [2], climate change evaluation [3], and pollution monitoring [4], etc. To accomplish these tasks safely and reliably, AUVs should realize accurate localization in their operating environments, which is one of their fundamental abilities.

\section{A. Simultaneous Localization and Mapping (SLAM)}

Traditionally, Doppler Velocity Logs (DVL) and Inertial Measurement Unit (IMU) data are fused with acoustic long [5], short [6] and ultra-short baseline[7] or GPS [8] for AUVs to localize their positions, which however require a prior knowledge of their environments. On the other hand, Simultaneous Localization and Mapping (SLAM) algorithm enables AUVs to fuse sensor data and build a map of unknown environment while localizing them in this built map simultaneously. The perception sensors used in underwater SLAM

The author Ling Chen, Aolei Yang are with the School of Mechatronics Engineering and Automation, Shanghai University, Shanghai 200072, China. Aolei Yang is the corresponding author(e-mail:aolei@shu.edu.cn). Huosheng $\mathrm{Hu}$ is with the School of Computer Science and Electronic Engineering, University of Essex, Colchester CO4 3SQ, U.K.(e-mail:hhu@essex.ac.uk). Wasif Naeem is with the School of Electronics, Electrical Engineering and Computer Science, Queen's University Belfast, University Road, Belfast, BT7 1NN, Northern Ireland, U.K. (email:w.naeem@qub.ac.uk). algorithms include underwater camera, multi-beam sonar, sidescan sonar and single-beam mechanically scanning imaging sonar (MSIS).

In general, the camera based visual SLAM algorithms conduct feature extraction and matching from the captured underwater images. Its front-end calculates relative camera motion between two either adjacent images or loop-closing images and its back-end conducts a bundle adjustment process to produce an optimized pose estimation [9][10]. The advantages of using underwater camera for SLAM include low cost, ability to provide rich information besides being easily deployed and interpreted based on recent advances in image/video processing. However, the cameras can only work in clean underwater environments with good light conditions for feature extraction. Therefore, there is limited literature available on using underwater cameras in visual SLAM algorithms in literature.

Both multi-beam sonar and side-scan sonar have been widely deployed on AUVs for safe operation and underwater exploration. More specifically, a multi-beam sonar emits multiple beams at different angles and obtain the range data from obstacles by analysing the returned multiple acoustic echoes. In contrast, a side scan sonar transmits the sound wave to the far side in a spherical wave after the sound pulse is emitted. After hitting the sea bottom, the reflected wave or the backscattered wave returns to the transducer along the original route. The intensity and time of the reflected wave can be detected and used to generate an image that represents the topography of the sea bottom.

The underwater images obtained by both multi-beam sonar and side-scan sonar are similar to the camera images and can be used for SLAM. For instance, Barkby et al. [11] proposed an EKF SLAM algorithm by fusing motion model and the result of scan matching of multi-beam sonar images. Chen et al. [12] implemented an EKF SLAM algorithm based on image feature points using side scan sonar. However, both multi-beam and side-scan sonar sensors are bulky, costly and power-consuming, which limit their applications on small AUV with a limited payload.

On the other hand, MSIS has become popular in small AUVs due to its low cost, small size, low power consumption and low computational complexity. However, it takes over 10 seconds to accomplish a complete scan (360 degrees). The scanned data is noisy and sparse, making the SLAM implementation difficult. To tackle these problems, various approaches have been proposed to parsing the image data 
obtained from MSIS to obtain the range-angle data for SLAM. Ribas et al. [13] and Dong et al. [14] used Hough Transform to extract line features by fitting the range-angle data from MSIS, and then feed them to the update phase of an EKF to realize accurate pose estimation of AUV.

\section{B. Rao-Blackwellized Partical Filter (RBPF)}

SLAM algorithms suffer from its enormous update complexity with an increasing number of features, a growing scale of environment and the linearisation error. Therefore, Burguera proposed two similar versions of localization algorithm based on scan matching, namely uspIC [15] and $\mu$ spIC [16]. A scan building module is first designed to gather a set of discretely sampled sonar reading points incorporating the vehicle motion estimated through EKF dead reckoning. Then the two consecutively built scans are registered to estimate the relative transform of these two scans, all of which are concatenated to produce the poses of AUV. However, matching consecutive scans is still likely to drift, resulting in unbounded localization errors as the vehicle travels.

Recently, particle Filter (PF) algorithms have been widely deployed in autonomous navigation of AUVs. The most popular one is Rao-Blackwellized PF (RBPF) [17]. Its basic idea is to utilize a set of particles to estimate the posterior of robot pose. Each particle represents a potential trajectory and an individual map is associated with each particle. This novel representation allows to cope with non-linear robot motion models of most autonomous robots. Therefore, RBPF SLAM have been successfully applied for building reliable occupancy grid maps for land-based mobile robots with laser range finders [18]. However, very few work have been conducted on utilizing RBPF to build occupancy grid map for AUVs with slow sampling MSIS.

This paper presents a novel RBPF SLAM algorithm for AUV equipped with a slow sampling sonar MSIS. It is able to build an accurate occupancy grid map while providing accurate estimation of AUV poses. The occupancy grid map can be used for global localization and path planning of AUV. The main contribution of the proposed algorithm is twofold: (1) A sliding-window based scan forming module is proposed to conquer scan distortion and sonar data sparseness caused by the slow-sampling MSIS; (2) A complete pipeline of applying RBPF SLAM is implemented to handle noisy and slowsampled sonar readings from MSIS and produce an accurate occupancy grid map.

The rest of the paper is organized as follows. Section II illustrates the problem of the slow-sampling characteristics of MSIS. The proposed RBPF SLAM algorithm for AUVs is detailed in Section III. Simulation and experiment results and analysis are given in Section IV to demonstrate the feasibility and performance of the proposed algorithm. Finally, a brief conclusion and future work are presented in Section V.

\section{The Slow-S Ampling Problem With MSIS}

A MSIS sonar is installed with a transducer head driven by a mechanical motor system for rotation at pre-set angular steps. It conducts scans in a horizontal 2D plane during the rotation

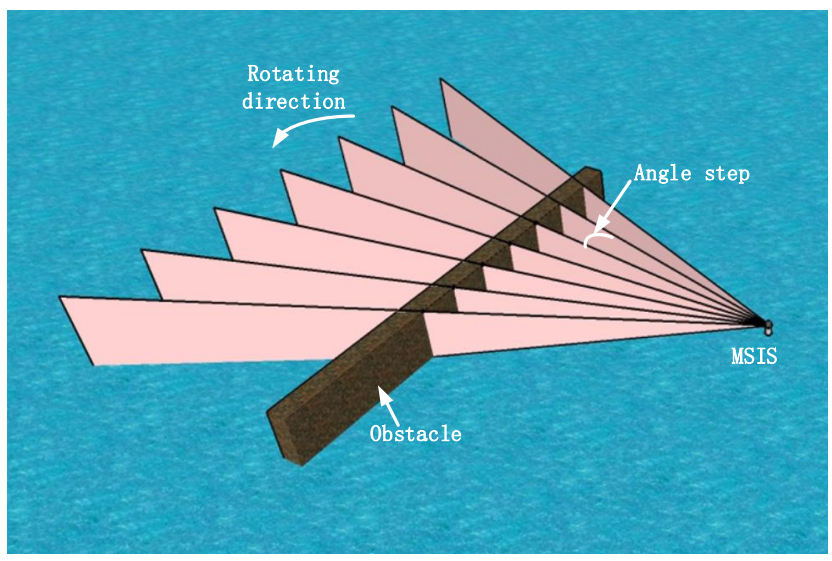

Fig. 1: A MSIS sensor is rotating its sonar head for collecting range data.

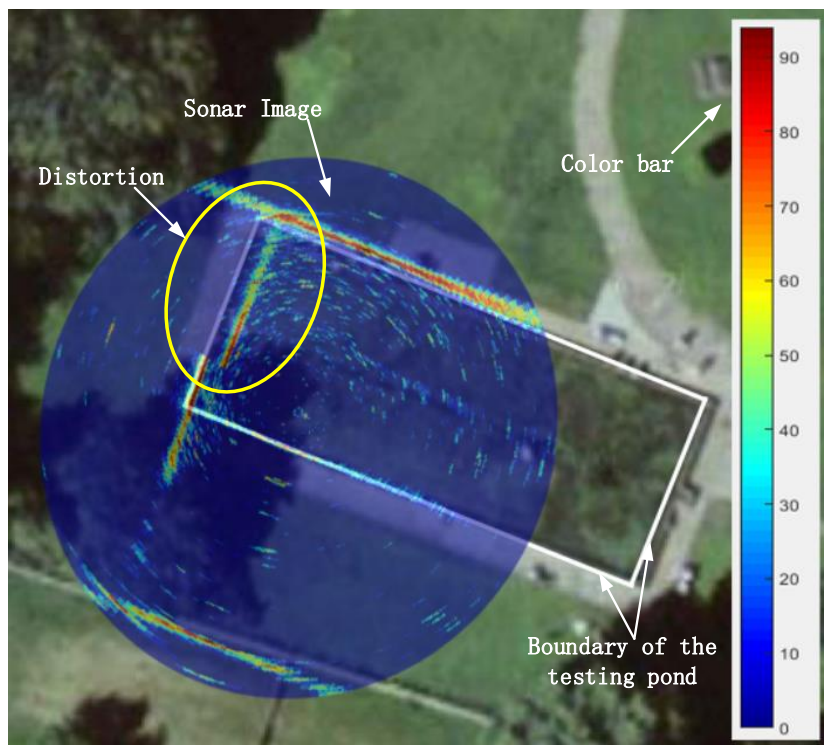

Fig. 2: A $360^{\circ}$ sonar image of a real rectangular testing pond. The color depth represents the echo amplitude of the sonar bins. As the AUV is moving while the image is generating, a distortion of the scan appears.

process. At each angular position, the MSIS head emits an acoustic beam which is fan-shaped with a narrow horizontal beam-width, as shown in Fig. 1. When this acoustic signal collides with any objects during its journey, it will return a certain energy whose amplitude determines the likelihood of objects. By measuring the time of flight of the returning signal, it can calculate the range using the speed of sound in water. Combining the amplitude of echo and calculated range, the sonar heads will generate a set of bins which can be visualized as an image shown in Fig. 2.

In our research, we have used a Tritech Micron sonar sensor that takes at least 6 seconds to accomplish one full $360^{\circ}$ scan, which may be even longer depending on the chosen parameters such as range, angle and step resolution of the sonar. If the 
AUV travels fast, the slowing scanning sonar will make the scanned image seriously distorted, as can be seen in Fig. 2. Also, its sparse data makes SLAM implementation difficult. The scan formation module based on a sliding window is proposed in this paper trying to tackle these challenging issues.

\section{The Proposed SLAM Algorithm}

Fig. 3 shows the architecture of the proposed SLAM algorithm. As can be seen, the algorithm is iterated by adopting a particle set of poses to guide the whole SLAM process. The dead reckoning module fuses data from a variety of sensors through an EKF to produce rough estimation of the AUV pose. It is used for scan forming process and the control input for propagating each pose particle.

The sonar reading queue $Q_{S R}$ is generated from the preprocessed MSIS by the beam segmentation module. The dead reckoning queue $Q_{D R}$ is used by the scan forming module to build a full 360-degree scan of segmented sonar readings. The reason for generating a full 360 degrees of sonar readings lies with the fact that the subsequent calculation of scan matching is more accurate and robust when a big angle range of scanned data is incorporated. The formed scan is then used for motion update and scan matching in the RBPF SLAM process.

Incorporating dead reckoning into the scan forming module is beneficial for getting rid of the sonar scan distortion resulted from the significant AUV motion and the slow sampling MSIS. Following the pipeline of sampling from dead reckoning, importance weighting, re-sampling and map estimation, the core part of the particle filter based SLAM module utilizes the formed scans $z_{t}$ and dead reckoning $u_{t}$ to estimate both AUV poses and a grid map of the environment iteratively. The detailed description of each module in the architecture is presented in the following sub-sections.

\section{A. Dead Reckoning}

Dead reckoning module aims to provide the rough estimation of the AUV pose using various sensors namely Inertial Measurement Unit, altimeter, Doppler Velocity Logger(DVL) if available. The dead reckoning module applies an EKF for pose estimation by fusing data from those sensors while the sonar is sampling data.

1) Process Prediction: Let the vehicle state $\boldsymbol{X}_{k}=$ $\left[\boldsymbol{p}_{k}^{T}, \boldsymbol{\varphi}_{k}^{T}\right]^{T}$ in the global coordinate frame be estimated at time $k$, where $\boldsymbol{p}_{k}$ represents the vehicle position, and $\boldsymbol{\varphi}_{k}$ represents the vehicle attitude of AUV. Specifically, $\boldsymbol{p}_{k}$ and $\varphi_{k}$ are defined as

$$
\boldsymbol{p}_{k}=\left[\begin{array}{lll}
x_{k} & y_{k} & z_{k}
\end{array}\right]^{T}, \quad \boldsymbol{\varphi}_{k}=\left[\begin{array}{lll}
\phi_{k} & \theta_{k} & \psi_{k}
\end{array}\right]^{T} .
$$

where $x_{k}, y_{k}, z_{k}$ are the position coordinates in each axis in the global coordinate frame and $\phi_{k}, \theta_{k}, \psi_{k}$ are euler angles roll, pitch and yaw in each corresponding axis.

Let the linear and angular velocities of AUV be $\boldsymbol{v}_{k}$ and $\boldsymbol{\omega}_{k}$, which are grouped to form as the control input $\boldsymbol{u}_{k}=$ $\left[\boldsymbol{v}_{k}^{T}, \boldsymbol{\omega}_{k}^{T}\right]^{T}$. Specifically, $\boldsymbol{v}_{k}$ and $\boldsymbol{\omega}_{k}$ are represented as

$$
\boldsymbol{v}_{k}=\left[\begin{array}{lll}
u_{k} & \nu_{k} & w_{k}
\end{array}\right]^{T}, \quad \boldsymbol{\omega}_{k}=\left[\begin{array}{lll}
o_{k} & q_{k} & r_{k}
\end{array}\right]^{T}
$$

where each element in the two vectors is the linear and angular velocity in each axis of the body frame of the AUV. Then, the process model for AUV can be expressed as a non-linear discrete time system:

$$
\boldsymbol{X}_{k+1}=f\left(\boldsymbol{X}_{k}, \boldsymbol{u}_{k}\right)=\boldsymbol{X}_{k}+\Delta_{T} J\left(\boldsymbol{X}_{k}\right) \boldsymbol{u}_{k}
$$

where

$$
J(\boldsymbol{X})=\left[\begin{array}{cccccc}
c \psi c \theta & c \psi s \theta s \phi-s \psi c \phi & c \psi s \theta c \phi+s \psi s \phi & 0 & 0 & 0 \\
s \psi c \theta & s \phi s \psi s \theta+c \psi c \phi & s \psi s \theta c \phi+s \phi c \psi & 0 & 0 & 0 \\
-s \theta & c \theta s \phi & c \theta c \phi & 0 & 0 & 0 \\
0 & 0 & 0 & 1 & s \phi t \theta & c \phi t \theta \\
0 & 0 & 0 & 0 & c \phi & -s \phi \\
0 & 0 & 0 & 0 & s \phi / c \theta & c \phi / c \theta
\end{array}\right]
$$

is the transformation matrix, and $\Delta_{T}$ is the sampling time interval. Note that, for simplicity, $s, c$ and $t$ in the above matrix are $\sin , \cos$ and tan functions, respectively.

If DVL is available, $\boldsymbol{u}_{k}$ can be represented by the measurement from DVL corrupted with an additive Gaussian noise $\boldsymbol{w}_{k} \sim \mathcal{N}\left(\mathbf{0}, Q_{u}\right)$. Whereas in the absence of DVL, $\boldsymbol{u}_{k}$ is assumed to be a Gaussian noise $\boldsymbol{w}_{k} \sim \mathcal{N}\left(\boldsymbol{C}, \boldsymbol{Q}_{\boldsymbol{u}}\right)$ with a constant mean $C$. As a result of this noise, the error of the dead reckoning will be accumulated and further unbounded. Thus, other sensor information is called in the update phase of EKF for correcting the error.

By virtue of (1), the vehicle state is estimated below:

$$
\hat{\boldsymbol{X}}_{k+1 \mid k}=f\left(\hat{\boldsymbol{X}}_{k \mid k}, \hat{\boldsymbol{u}}_{k}\right)
$$

and the covariance matrix of the prediction error is provided by:

$$
\mathbf{P}_{k+1 \mid k}=\mathbf{F}_{k+1} \mathbf{P}_{k \mid k} \mathbf{F}_{k+1}^{\mathrm{T}}+\mathbf{G}_{k+1} \boldsymbol{Q}_{\boldsymbol{u}} \mathbf{G}_{k+1}^{\mathrm{T}}
$$

where $\mathbf{F}_{k+1}$ and $\mathbf{G}_{k+1}$ are the Jacobian matrices formed by taking the partial derivatives of the non-linear model function $f$ with respect to the state $\hat{\boldsymbol{x}}_{k}$ and the noise $\boldsymbol{w}_{k}$, respectively.

2) Update with Altimeter: The depth of the AUV in underwater can be used for providing the $z_{k}$ measurement in $\boldsymbol{p}_{k}$, and the depth measurement equation can be expressed as

$$
z_{d, k}=\mathbf{H}_{d, k} \boldsymbol{X}_{k}+\sigma_{d, k}=\left[\begin{array}{llll}
0 & 0 & 1 & \mathbf{0}_{1 \times 3}
\end{array}\right] \boldsymbol{X}_{k}+\sigma_{d, k}
$$

where $\sigma_{d, k}$ is a zero-mean Gaussian noise with the covariance being $R_{d}$.

3) Update with Orientation from IMU: The IMU is able to provide the orientation measurement whose model can be expressed as:

$$
\boldsymbol{z}_{a, k}=\mathbf{H}_{a, k} \boldsymbol{X}_{k}+\boldsymbol{\sigma}_{a, k}=\left[\begin{array}{ll}
\mathbf{0}_{3 \times 3} & \boldsymbol{I}_{3 \times 3}
\end{array}\right] \boldsymbol{X}_{k}+\boldsymbol{\sigma}_{a, k}
$$

where $\boldsymbol{I}$ represents the $3 \times 3$ identity matrix and $\boldsymbol{\sigma}_{a, k}$ is a zeromean Gaussian noise with the covariance being $\boldsymbol{R}_{a}$. Then the model prediction is updated by applying standard EKF update equations to produce the dead reckoning estimation.

\section{B. Scan Forming based on a Sliding Window}

The scan forming module can apply the results of dead reckoning to form a full $360^{\circ}$ scan as if all scanned points are read instantaneously. A sliding window strategy is employed to govern the scan forming. Two queues, namely $Q_{S R}$ and $Q_{D R}$, are used to maintain both the history of dead reckoning and sonar reading. The size of the queue is equal to the number of the scanned sonar points of a full $360^{\circ}$ scan. When a new 


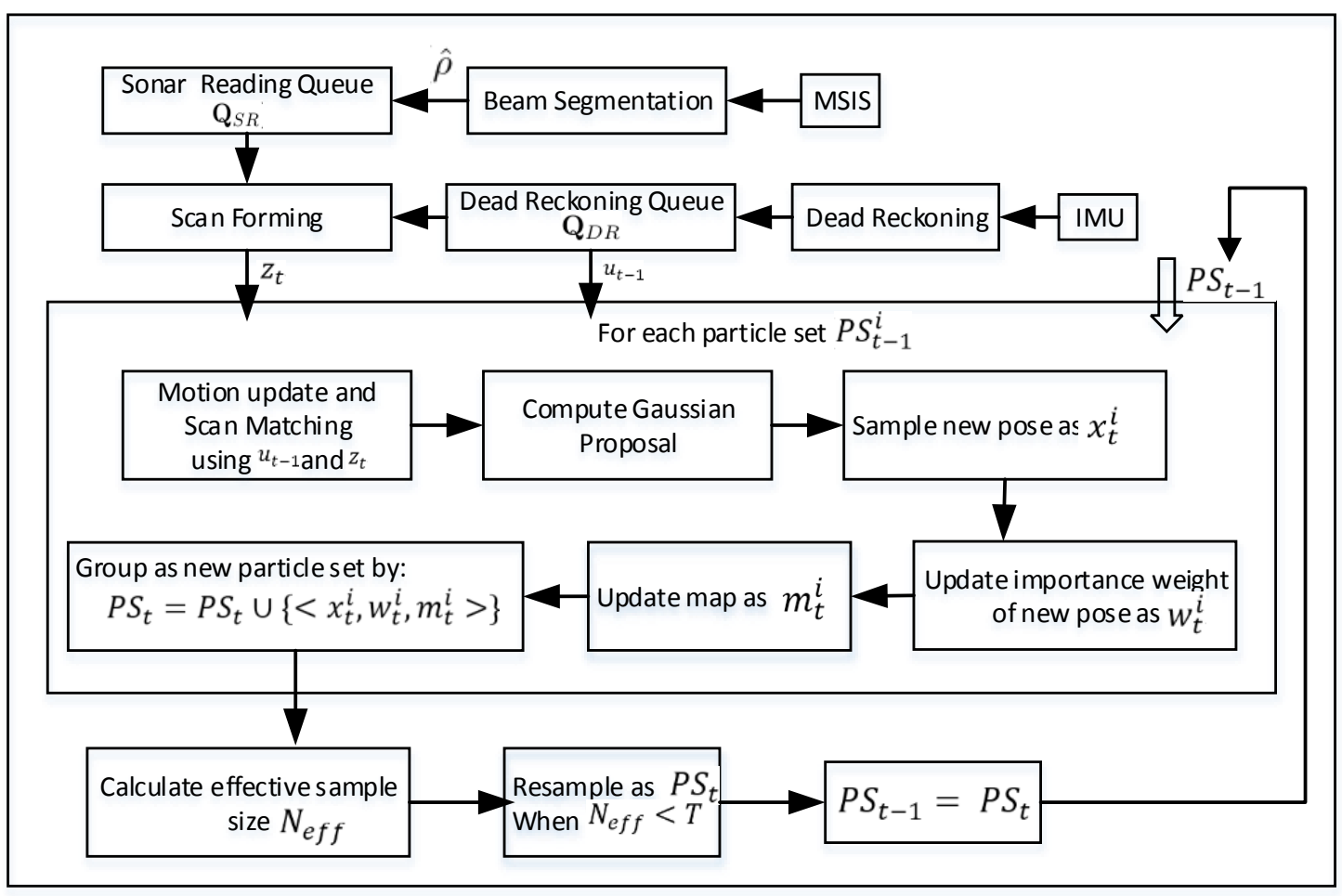

Fig. 3: The architecture of the proposed algorithm.

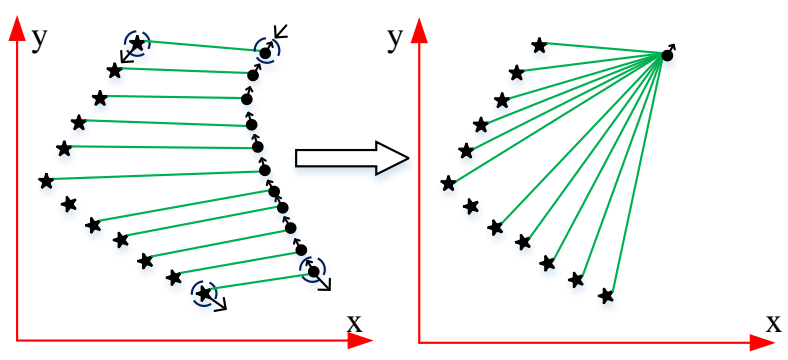

Fig. 4: The diagram of the scan forming process based on the sliding window strategy.

sonar point is obtained, it is pushed back into the rear of $Q_{S R}$ while the front one is pushed out. Simultaneously, the dead reckoning pose corresponding to the new sonar point is also pushed back into the rear of $Q_{D R}$ while the front one is pushed out.

This strategy deploys historical sonar readings to solve data deficiency caused by the slow sampling MSIS. Fig. 4 shows the scan forming process that utilizes a sliding window strategy, in which the black stars represent the preprocessed sonar readings and the black dots represent the dead reckoning poses.
Each sonar point $P_{k, i}^{S}$ in the queue of $Q_{S R}$ is transformed from the current robot body frame $\left\{R_{k}\right\}$ to the previous robot body frame, namely $\left\{R_{l}\right\}$. This means that all the scanned points will be represented in frame $\left\{R_{l}\right\}$, as shown in Fig. 5 . The transformation is based on the fact that the sonar reading is kept the same in the world coordinate frame $\{W\}$, which can be described by the following equation:

$$
\hat{P}_{i}^{W}=\hat{\mathbf{T}}_{R_{l}}^{W} \oplus \mathbf{T}_{S}^{R_{l}} \oplus P_{l, i}^{S}=\hat{\mathbf{T}}_{R_{k}}^{W} \oplus \mathbf{T}_{S}^{R_{k}} \oplus P_{k, i}^{S}
$$

where $\hat{P}_{i}^{W}$ is the $i$ th sonar reading with respect to the world frame $\{W\}, \hat{\mathbf{T}}_{R_{l}}^{W}$ is the last transform of the robot body frame $\left\{R_{l}\right\}$ with respect to the world frame $\{W\}$ and the similar representation goes to $\mathbf{T}_{S}^{R_{l}}, \hat{\mathbf{T}}_{R_{k}}^{W}, \mathbf{T}_{S}^{R_{k}}$ with $\{S\}$ being the sensor frame and $\left\{R_{k}\right\}$ being the $k$ th robot body frame.

As the transform from the sensor frame to the robot body frame is static, we have $\mathbf{T}_{S}^{R_{l}}=\mathbf{T}_{S}^{R_{k}}$. From equation (6), the point $P_{k, i}^{S}$ can be represented with respect to the body frame of the last sonar $\left\{R_{l}\right\}$ as:

$$
\hat{P}_{l, i}^{S}=\ominus \mathbf{T}_{S}^{R_{l}} \ominus \hat{\mathbf{T}}_{R_{l}}^{W} \oplus \hat{\mathbf{T}}_{R_{k}}^{W} \oplus \mathbf{T}_{S}^{R_{k}} \oplus P_{k, i}^{S}
$$

where $\oplus$ and $\ominus$ are the compounding and inversion transformations proposed in [19].

Algorithm 1 describes the scan forming process based on the sliding window. The input parameters are the new sonar reading $\hat{\rho}$, covariance of the sonar reading $\mathbf{P}_{\rho}$, current dead reckoning $\boldsymbol{X}_{k}$, last dead reckoning $\boldsymbol{X}_{k-1}$, current estimated robot pose $\hat{\boldsymbol{X}}_{k}$, the queue of dead reckoning $Q_{D R}$ and the queue of sonar reading $Q_{S R}$. The formed scan is denoted as 


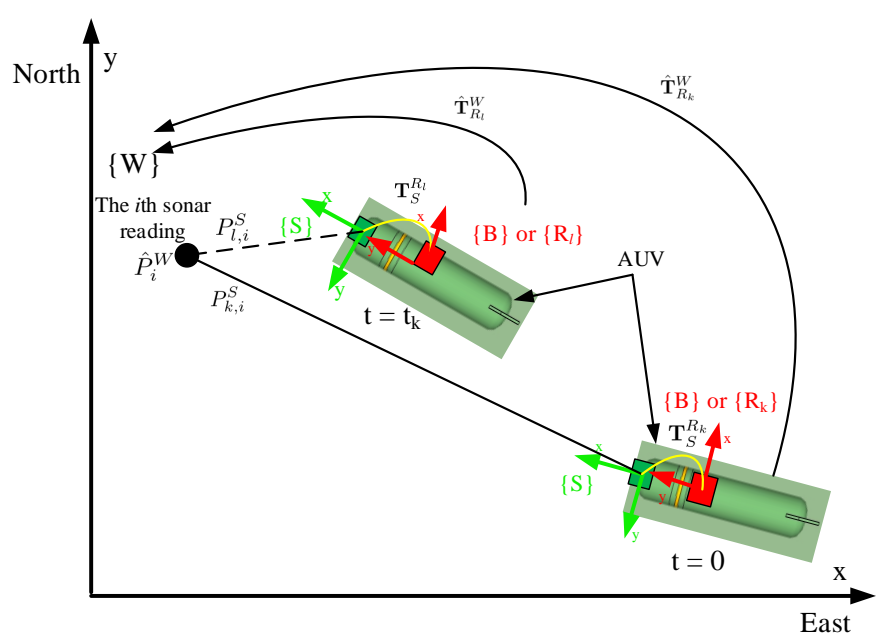

Fig. 5: The representation of different coordinate systems during scan forming.

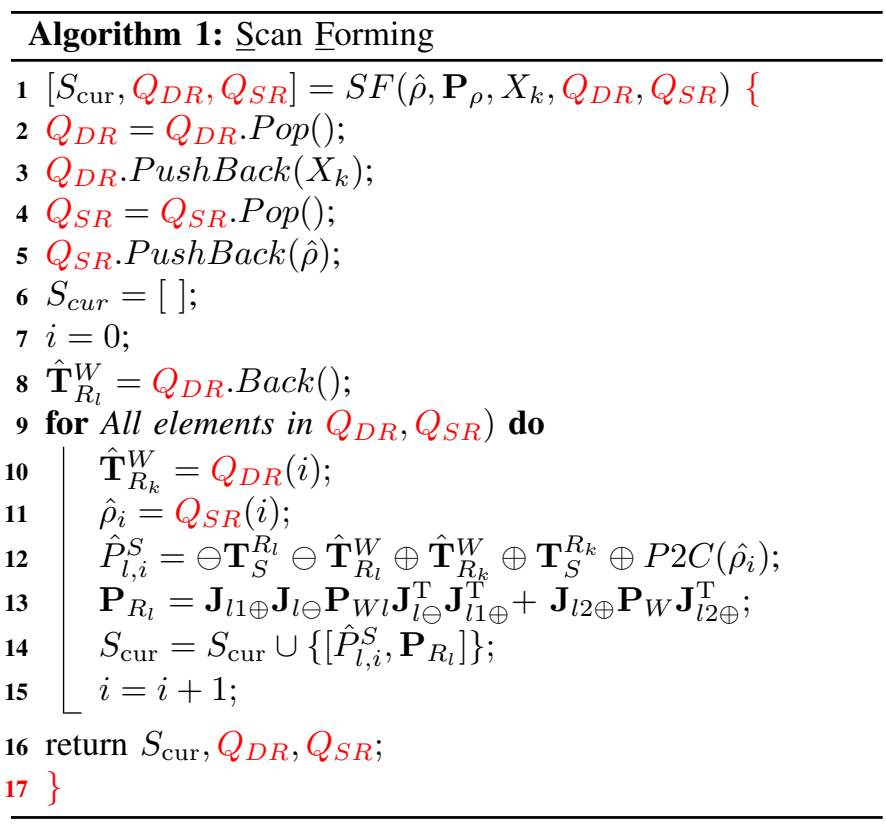

$S_{\text {cur }}$ which is also the returned value of the algorithm. $\mathbf{P}_{W}$ is the covariance of current sonar reading represented in global frame $\{W\}, \mathbf{J}_{l 1 \oplus}, \mathbf{J}_{l \ominus}$ and $\mathbf{J}_{l 2 \oplus}$ are the Jacobian matrices of $\hat{P}_{l, i}^{S}$ with respect to $\hat{\mathbf{T}}_{R_{k}}^{W}$ and $\hat{\mathbf{p}}_{\mathbf{i}}^{\mathbf{W}}$.

\section{RBPF SLAM with MSIS}

The whole flowchart of the proposed algorithm is presented in Algorithm 2. $A$ is the size of the queue for both $Q_{S R}$ and $Q_{D R}$. After a full scan $S_{c u r}$ has been formed by abovementioned Algorithm 1, it is then considered as a measurement $z_{t}$ and fed into a RBPF SLAM pipeline. The SLAM problem is solved by factorizing the SLAM process into separate localization and mapping parts, which stems from the theory that a joint probability can be converted to the product of conditional probability:

$p\left(x_{1: t}, m \mid z_{1: t}, u_{1: t-1}\right)=p\left(m \mid x_{1: t}, z_{1: t}\right) \cdot p\left(x_{1: t} \mid z_{1: t}, u_{1: t-1}\right)$

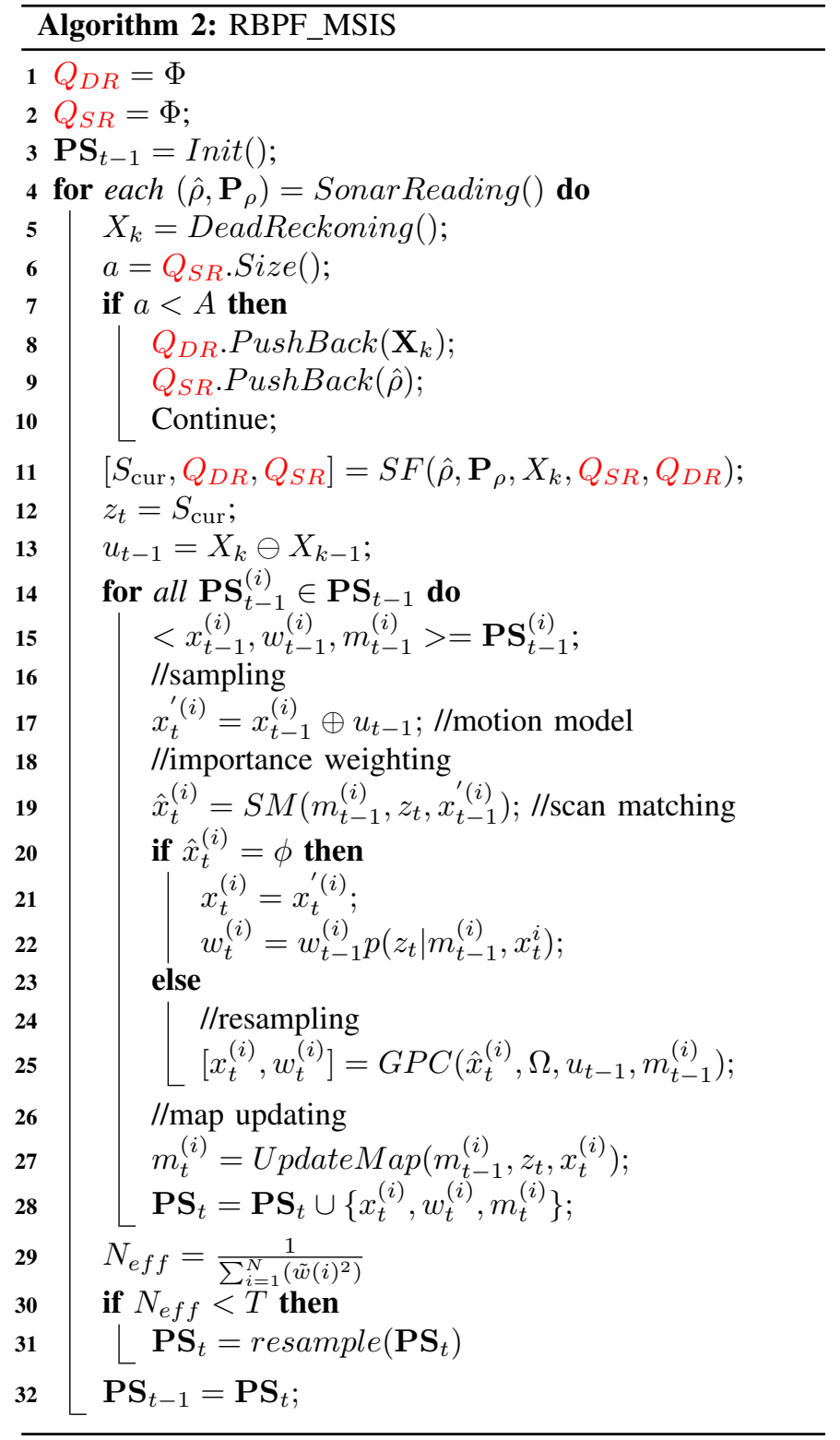

where $p\left(x_{1: t} \mid z_{1: t}, u_{1: t-1}\right)$ is the posterior of about potential trajectories $x_{1: t}$ of the robot given its observations $z_{1: t}$ and its odometry measurements $u_{1: t}, p\left(m \mid x_{1: t}, z_{1: t}\right)$ is the posterior over maps, and $p\left(x_{1: t}, m \mid z_{1: t}, u_{1: t-1}\right)$ is the posterior posterior over maps and trajectories. As $p\left(m \mid x_{1: t}, z_{1: t}\right)$ can be computed analytically [15] given the knowledge of $x_{1: t}$ and $z_{1: t}$, the key is to compute $p\left(x_{1: t} \mid z_{1: t}, u_{1: t-1}\right)$.

To estimate the posterior $p\left(x_{1: t} \mid z_{1: t}, u_{1: t-1}\right)$, a set of particles are utilized. Each particle is composed of robot pose $x$, weight $w$ and the grid map $m$. Then the particle filter algorithm incrementally utilizes the available dead reckoning and sonar scan data to update the set of particles which represents the posterior over the map and the trajectory of the AUV. The process includes four steps: sampling, importance weighting, resampling and map updating [18]. The function $\hat{x}_{t}^{(i)}=$ $S M\left(m_{t-1}^{(i)}, z_{t}, x_{t-1}^{(i)}\right)$ stands for scan matching and is used for finding the most likely pose that matches against the current map $m_{t-1}^{(i)}$ with current observation $z_{t}$ and $x_{t-1}^{(i)}$ being the 


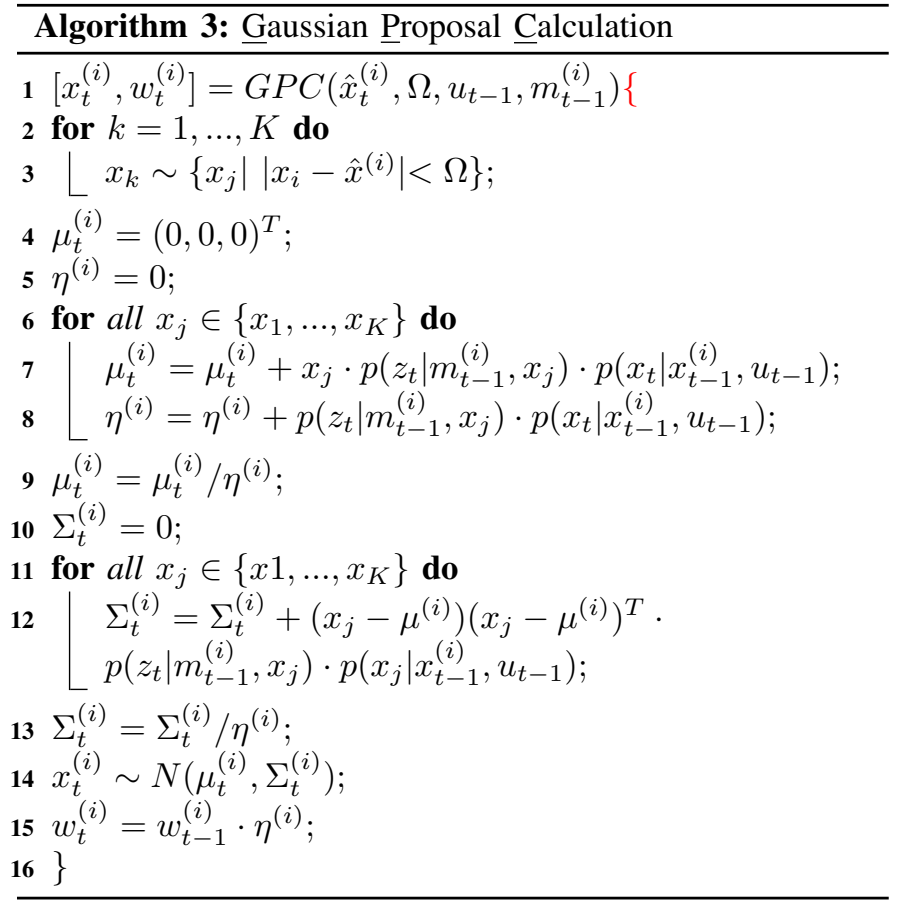

initial estimation [20].

Algorithm 3 shows the Gaussian proposal calculation pipeline which aims to calculate the Gaussian approximation of the proposal distribution, based on which the new particle is sampled for next iteration. $\Omega$ is the interval threshold for selecting the points around the neighbouring area of $\hat{x}_{t}^{(i)}$. The likelihood of an observation based on the map and pose $p\left(z_{t} \mid m_{t-1}^{(i)}, x_{j}\right)$ is computed by using a "beam endpoint model" proposed by [21] instead of "full beam model". The reason is twofold: 1) the computational complexity of "full beam model" is much higher than that of "beam endpoint model"; 2) as there are not many dynamic obstacles in our testing scenarios, the "beam endpoint model" becomes unnecessary. The term $p\left(x_{j} \mid x_{t-1}^{(i)}, u_{t-1}\right)$ can be calculated using the odometry motion model proposed in [22]. The map $m_{t}^{(i)}$ is then updated with the drawn pose $x_{t}^{(i)}$ and the observation $z_{t}$. The map and pose of the particle with the biggest weight are then chosen as the built map and the estimated AUV pose.

\section{EXPERIMENTAL RESUlts}

Both simulation and practical experiments are conducted with two different AUVs and testing sites to verify the effectiveness of the proposed RBPF-MSIS algorithm. Table I shows the main parameters of our algorithm for both simulation and practical experiments.

TABLE I: The parameters of the RBPF-MSIS algorithm

\begin{tabular}{ll}
\hline Parameter & Value \\
\hline Map resolution & $0.25 \mathrm{~m}$ \\
Number of particles & 120 \\
Resample threshold, $T$ & 0.5 \\
Interval threshold for selecting points & $1.5 \mathrm{~m}$ \\
around the estimated pose $\hat{x}_{t}^{(i)}, \Omega$ & \\
The number of iterations of the scan matcher & 5 \\
\hline
\end{tabular}

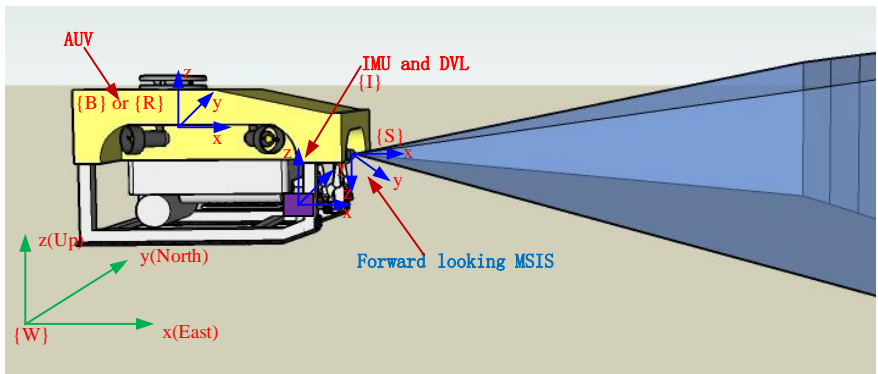

Fig. 6: Simulation set-up for our AUV.

\section{A. Simulation}

1) Simulation Set-up: Fig. 6 shows the simulation set-up for our AUV. As can be seen, our AUV is equipped with various sensors such as IMU, DVL(frame is denoted as $\{I\}$ ) and a forward looking MSIS(frame is denoted as $\{S\}$ ). The AUV body frame is represented with $\{B\}$ or $\{R\}$ while the global frame is denoted as $\{W\}$ whose axis $x$ points to East, axis $y$ to North and axis $z$ to the up direction.

Fig. 7 shows the simulation environment which is an anomalous-shaped pond where the AUV is controlled to navigate around. The 3D model of the environment is loaded into the 3D simulator Gazebo [23]. Robot Operating System (ROS) [24] is used in our simulation for convenience in interfacing with Gazebo. Drivers for IMU, DVL and MSIS are loaded as plug-ins of Gazebo for publishing ROS-compatible data which is subscribed by the proposed SLAM algorithm. During the simulation, our AUV is controlled to navigate around the middle object for one round. The speed of the AUV is set as $0.1 \mathrm{~m} / \mathrm{s}$ which is added by a Gaussian noise whose standard variance is $0.02 \mathrm{~m} / \mathrm{s}$. The outputs of the algorithm include an occupancy grid map and an estimated AUV trajectory.

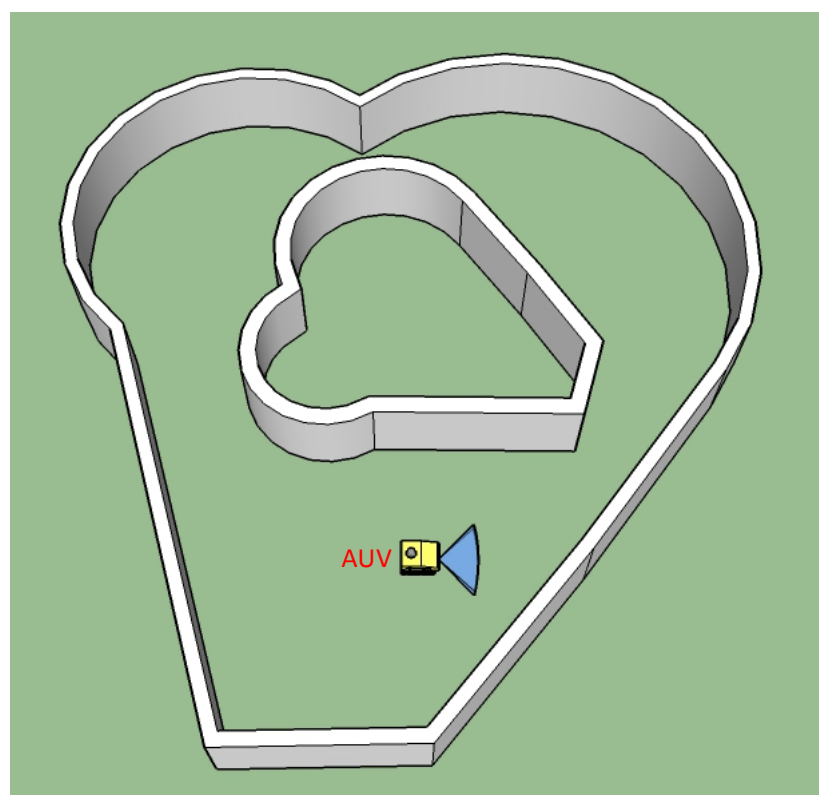

Fig. 7: The simulated environment for AUV manoeuvring. 
2) Simulation Result: To verify the effectiveness of the proposed algorithm, we implemented different SLAM algorithms in simulation, e.g. no scan forming incorporating vehicle motion, normal scan forming and sliding-window-based scan forming. Fig. 8, Fig. 9 and Fig. 10 show the occupancy grid maps and trajectories produced by these algorithms. The green line indicates the ground truth trajectory while the blue line represents the dead reckoning trajectory. The trajectories generated by each algorithm are represented with different colors, with the red one being SLAM trajectory without scan forming incorporating motion, the cyan one being the SLAM trajectory with normal scan forming, and the purple one being the SLAM trajectory with sliding-window-based scan forming.

In contrast to sliding-window-based scan forming, normal scan forming is the process of forming a full scan after the whole set of $360^{\circ}$ sonar readings have been collected. It can be seen from the Fig. 8 that the occupancy grid map produced by the SLAM algorithm which does not use scan forming incorporating motion exhibits serious ghost shadow and is inconsistent with the environment.

Thanks to the scan forming process which incorporates vehicle motion, Fig. 9 shows the occupancy grid map generated by the SLAM algorithm with normal scan forming, which has much smaller ghost effect than Fig. 8. Since the sliding-window-based scan forming takes the advantages of the history sonar data sufficiently, the occupancy grid map built by the SLAM algorithm with sliding-window-based scan forming has the smallest ghost shadow and matches best with the environment. As shown in Fig. 10, the trajectory produced by the SLAM with sliding-window-based scan forming is closest to the ground truth, compared with trajectories given by the SLAM algorithms with normal scan forming and without scan forming.

Fig. 11 shows the deviation of trajectories by different algorithms from the ground truth in detail. The deviation is represented by the Euclidean relative localization error between the trajectories produced by above-mentioned different SLAM algorithms and the ground truth. It is clear that the estimation error of dead reckoning (blue star line) is the largest due to accumulated integration error caused by sensor noises. The distance error of SLAM with scan forming (SF) without incorporating motion (magenta circle line) is smaller than dead reckoning, but it is still far from ideal.

The distance error of SLAM with normal scan forming and motion correction (MC) is smaller than the errors of the SLAM without scan forming and dead reckoning. In contrast, the SLAM with sliding-window-based scan forming with MC (red point line) has the smallest distance error. The root mean squared error (RMSE) proposed in [25] for dead reckoning, SLAM with scan forming (SF) without MC, SLAM with normal scan forming with MC and SLAM with slidingwindow-based scan forming with $\mathrm{MC}$ are evaluated to be $6.47 \mathrm{~m}, 3.74 \mathrm{~m}, 1.67 \mathrm{~m}$ and $1.02 \mathrm{~m}$, respectively. Therefore, both the distance error and translational RMSE indicate that the proposed SLAM algorithm with sliding-window-based scan forming with MC produces the most accurate pose estimation.

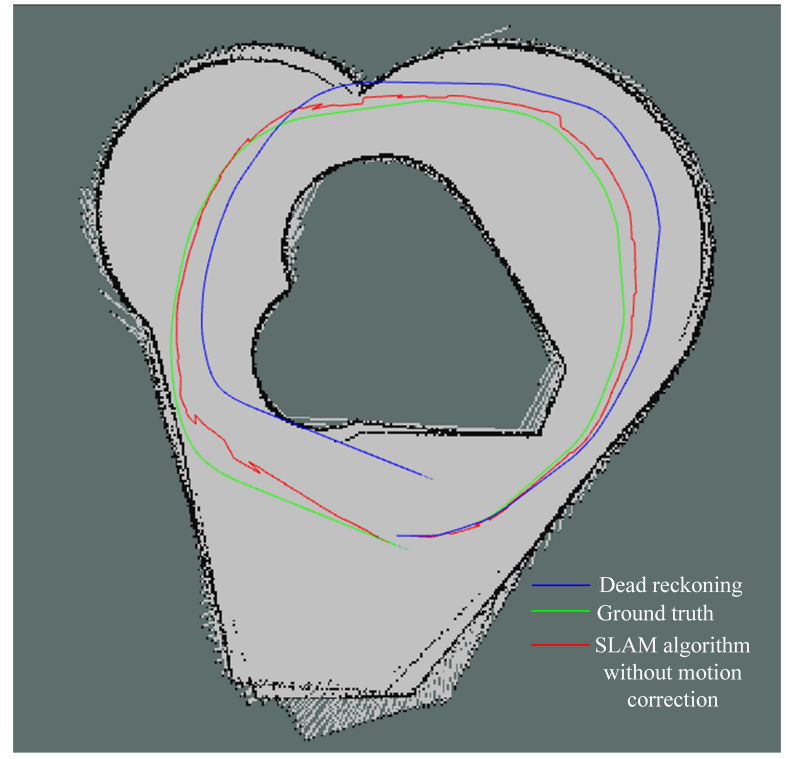

Fig. 8: Occupancy grid map and trajectories produced by SLAM algorithm with scan forming without incorporating motions.



Fig. 9: Occupancy grid map and trajectories produced by SLAM algorithm with normal scan forming incorporating motions.

\section{B. Experiments with a home-made AUV}

To further examine the proposed SLAM algorithm, we conducted practical experiments using a home-made AUV, which is named as SCPBot and shown in Fig. 12a. An embedded system is deployed on our AUV to collect sensor data from IMU and MSIS. The MSIS is a product named as Micron DST from the Tritech International Limited. IMU is used for providing 3D orientation measurement. The MSIS is controlled to rotate continuously in the clockwise scanning direction (from $0^{\circ}$ to $360^{\circ}$ ) to sample sonar data. The sampling range of MSIS is set as $20 \mathrm{~m}$ and the rotation step angle is configured as $1.8^{\circ}$. 


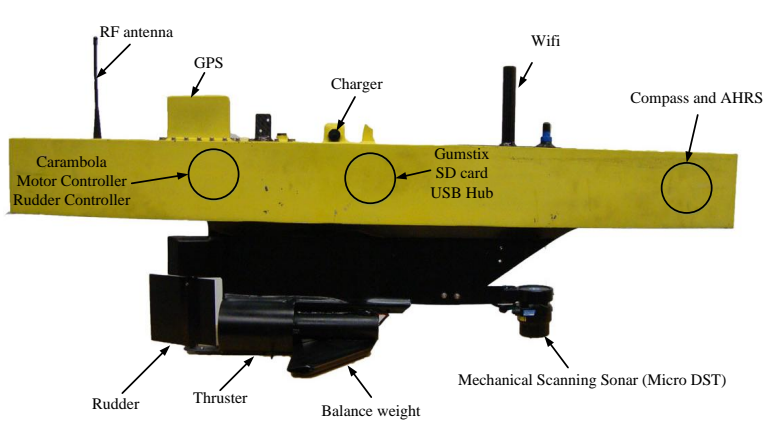

(a) The AUV platform: SCPBot.

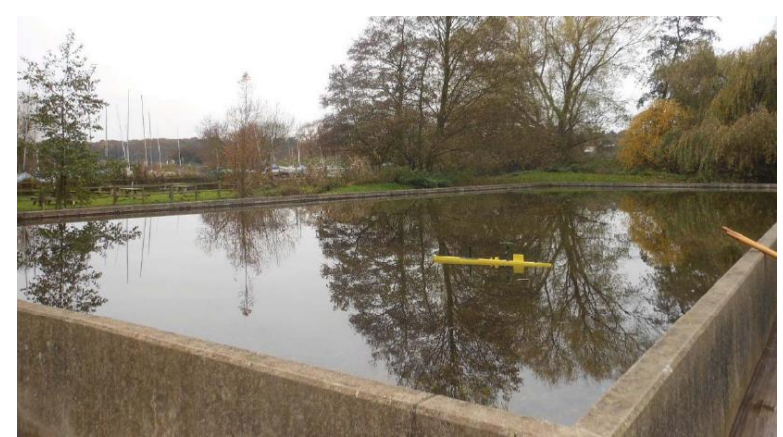

(b) Vehicle gathering data in a rectangular pond.

Fig. 12: The testing site for our home-made underwater vehicle which is gathering data.

We use a joystick to send control signals to our AUV via its wireless antenna above the water surface so that the vehicle can be manoeuvred remotely for data collection. The testing site is a rectangular pond shown in Fig. 12b where the vehicle is tele-operated to swim for 2 cycles. As the GPS signal is not accurate enough, the starting position and the stopping position is deliberately controlled to be the same in order to verify the accuracy of localization. Preprocessed by using dynamic threshold based beam segmentation algorithm proposed by [13], the sonar readings together with IMU data are imported into the proposed RBPF-SLAM algorithm pipeline.

Fig. 13 presents the effect of scan forming, in which the sonar scans (blue dot) coincides with the boundary of the testing site. It is clear that the scan forming strategy can effectively eliminate the distortion (pink diamond) caused by the slow-sampling MSIS.

Fig. 14 shows the the occupancy grid map rendered with the poses estimated by uspIC [15] and the map built by the proposed algorithm. It can be seen that the occupancy grid map rendered by the uspIC is seriously distorted and inconsistent with the rectangular pond wall due to the pose estimation error. The occupancy grid map built by the proposed SLAM algorithm matches well with the real rectangular pond wall. Although DGPS can be used to obtain absolute ground truth position of the AUV, it was not available for our testing as the base station is far away from the receiver on our AUV.

Therefore, we used the distance error between the start position and end position of the trajectory to evaluate the accuracy of different algorithms. The relative localization error between the start position and the end position of the trajectory estimated by dead reckoning, uspIC and the proposed RBPFSLAM algorithm were $7.77 \mathrm{~m}, 4.37 \mathrm{~m}$, and $1.29 \mathrm{~m}$, respectively. This further verified that the proposed RBPF-SLAM algorithm provides the most accurate pose estimation.

\section{Experiment Using a Real Dataset in [13]}

To extensively validate the effectiveness of the proposed algorithm, external data obtained by Ribas [13] is also used for validation. Ictineu AUV developed in University of Girona, as shown in Fig. 15a, was used to gather data in an abandoned marina located near St. Pere Pescador in the Costa Brava,
Spain (see Fig. 15b). The AUV was tele-operated to maneuver at an average speed of $0.2 \mathrm{~m} / \mathrm{s}$ and the whole trajectory is 600 meters. The experiment lasted 55 minutes 44 seconds.

The trajectory comprises a small loop and a long straight path with 200 meters. The data set contained measurements from a DVL, a compass, a Motion Reference Unit(MRU) and MSIS. The MSIS was configured as a sampling range of 50 meters, a resolution of 0.1 meter and step angle of 1.8 degrees. In addition, a buoy with a DGPS was equipped on the robot for obtaining the ground truth.

Similar to Fig. 14, Fig. 16 shows the occupancy grid map built by uspIC and the proposed algorithm. Similar to Fig. 11, Fig. 17 shows the relative localization errors among the uspIC trajectories, the proposed SLAM algorithm and the ground truth. It can also be seen clearly that the proposed algorithm outperforms the uspIC in terms of the level of map consistency with the real environment and the pose estimation accuracy.

\section{Real Time Performance}

The time to obtain the dataset for both SCPBot and Ictineu AUV and the time to run the proposed algorithm RBPF-MSIS is listed in Table II. The algorithm is run on a normal laptop PC with Intel i5 CPU at $2.3 \mathrm{GHz}$ and 8GB memory. As can be seen from the table, the time to run the algorithm is much faster than the time to obtain the dataset. On the other hand, the time to execute RBPF-MSIS is smaller than the time to run uspIC, which indicates that the computational complexity of RBPF-MSIS is lower than that of uspIC.

Fig. 18 shows the time for running RBPF-MSIS algorithm after each sonar reading has been received during a full 360 degree of scan with SCPBot AUV. It can be seen that the execution time for the proposed algorithm for each sonar reading is much less than the time span $(43 \mathrm{~ms})$ for two consecutive sonar readings, and the average running time is 11 ms. Therefore, both Table II and Fig. 18 demonstrate that sonar data can be processed before a new data is received, and the proposed algorithm is suitable for online applications.

\section{CONCLUSiOn AND Future Work}

This paper presents a novel SLAM algorithm for AUV equipped with a MSIS sonar sensor that samples data at a 


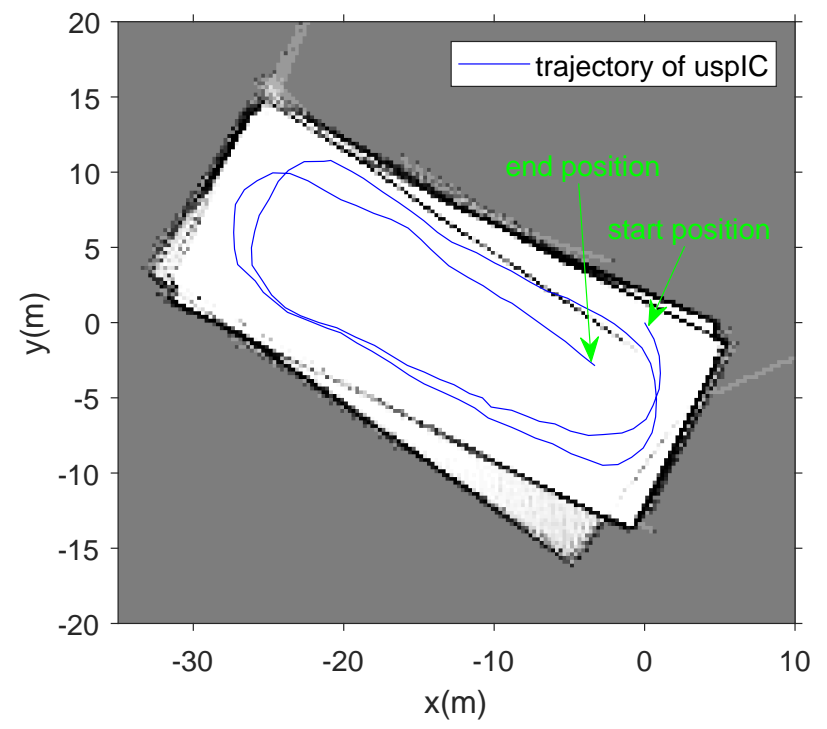

(a) Occupancy grid map rendered with poses estimated by uspIC

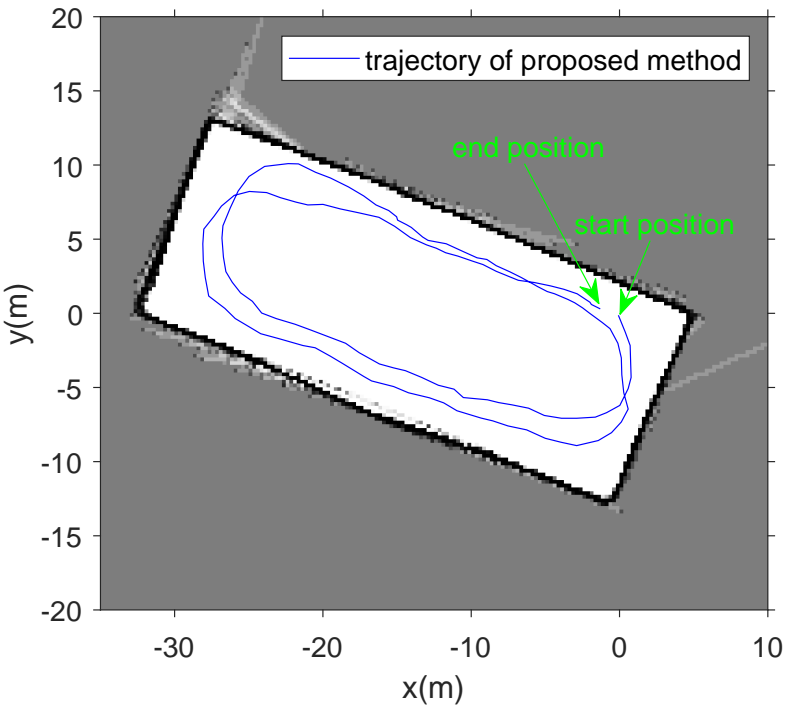

(b) Occupancy grid map built by the proposed algorithm.

Fig. 14: Occupancy grid maps rendered with poses estimated by uspIC and built by the proposed algorithm.

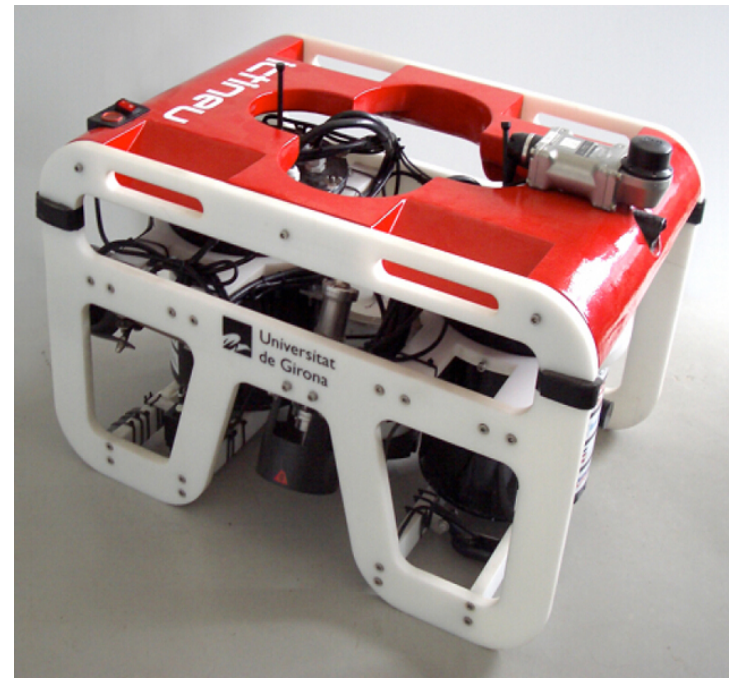

(a) The Ictineu AUV developed in the University of Girona.

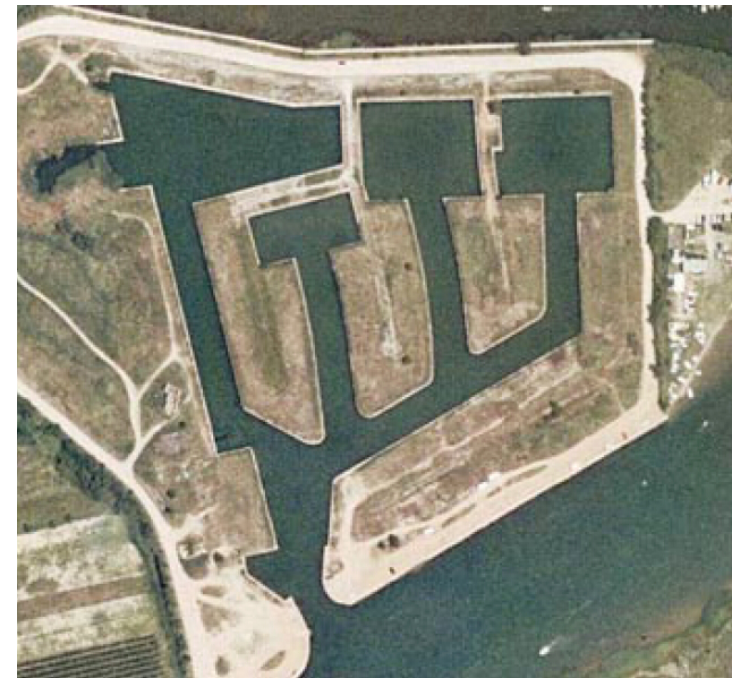

(b) The place where data was collected

Fig. 15: The Ictineu AUV and the place where data was collected [13].

TABLE II: Computation time of the RBPF-MSIS algorithm for experimental platforms

\begin{tabular}{lll}
\hline & SCPBot & Ictineu AUV \\
\hline Time to obtain dataset & $17 \mathrm{~min} 10 \mathrm{~s}$ & $55 \mathrm{~min} 44 \mathrm{~s}$ \\
Time to run RBPF-MSIS & $4 \mathrm{~min} 20 \mathrm{~s}$ & $14 \mathrm{~min} 6 \mathrm{~s}$ \\
Time to run uspIC & $6 \mathrm{~min} 40 \mathrm{~s}$ & $20 \mathrm{~min} 10 \mathrm{~s}$ \\
\hline
\end{tabular}

slow rate. The algorithm is based on RBPF and a carefully designed sliding window based scan forming module. It is able to form a complete scan each time when a single sonar reading data is obtained by incorporating the history of sonar readings. In this way, the distortion and data sparseness caused by the slow-sampling MSIS can be tackled effectively. With the generated scans, the proposed SLAM algorithm is able to produce consistent occupancy-grid maps and localize the AUV accurately. Extensive simulation and real experiment results show that the proposed algorithm outperformed other existing localization algorithm in terms of map consistency and localization accuracy.

It should be noticed that the proposed SLAM algorithm was tested off-line by using the dataset of Ictineu AUV during the real experiments. Therefore, our future work will focus on implementing the proposed algorithm on-line by using real data obtained by an AUV that operates in real world missions.

\section{ACKNOWLEDGEMENTS}

This research has been financially supported by $\mathrm{Na}$ tional Natural Science Foundation of China (Grant number 61703262 and 61873158), Shanghai Sailing Program 


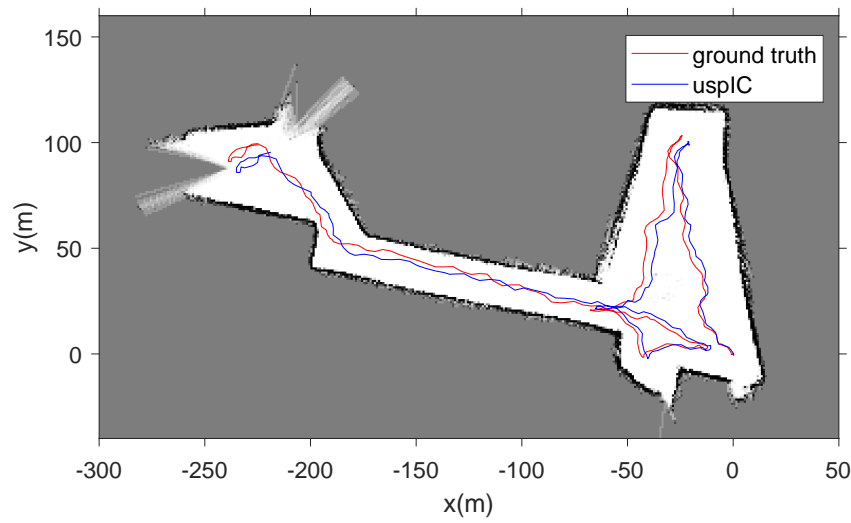

(a) Occupancy grid map rendered with poses estimated by uspIC.

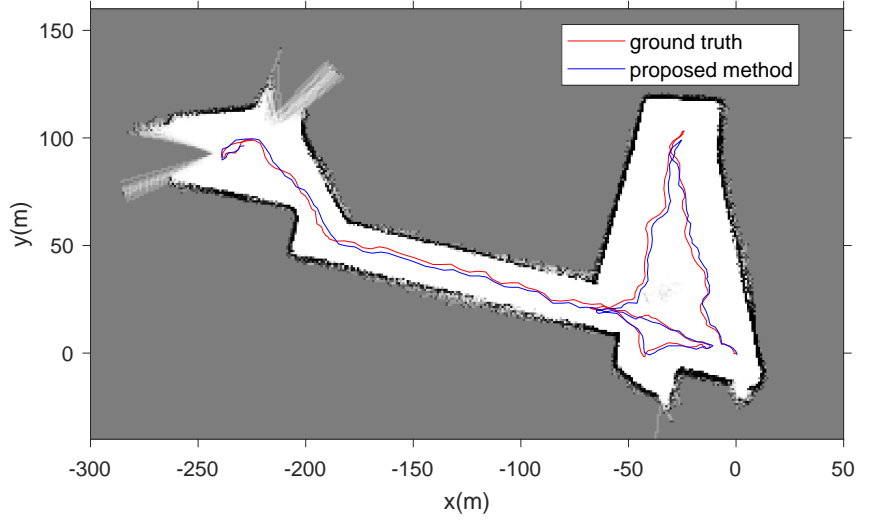

(b) Occupancy grid map built by the proposed algorithm.

Fig. 16: Occupance grid maps rendered with the poses estimated by uspIC and the proposed algorithm using dataset in [13].

(16YF1403700), and the Natural Science Foundation of Shanghai (18ZR1415100). Our thanks also go to Robin Dowling and Ian Dukes for their technical support during the experiments, and to David Ribas et al. for making their dataset of Ictineu AUV available publicly.

\section{REFERENCES}

[1] S. Venkatesan, "AUV for Search and Rescue at sea - an innovative approach," in 2016 IEEE/OES Autonomous Underwater Vehicles (AUV), Nov 2016, pp. 1-9.

[2] D. Thompson, D. Caress, H. Thomas, and D. Conlin, "MBARI mapping AUV operations in the gulf of California 2015," in OCEANS 2015 MTS/IEEE Washington, Oct 2015, pp. 1-7.

[3] S. B. Williams, O. R. Pizarro, M. V. Jakuba, C. R. Johnson, N. S. Barrett, R. C. Babcock, G. A. Kendrick, P. D. Steinberg, A. J. Heyward, P. J. Doherty et al., "Monitoring of benthic reference sites: using an autonomous underwater vehicle," IEEE Robotics Automation Magazine, vol. 19, no. 1, pp. 73-84, 2012.

[4] A. Tonacci, G. Lacava, M. A. Lippa, L. Lupi, M. Cocco, and C. Domenici, "Electronic nose and AUV: A novel perspective in marine pollution monitoring," Marine Technology Society Journal, vol. 49, no. 5, pp. 1824, 2015.

[5] A. Matos, N. Cruz, A. Martins, and F. L. Pereira, "Development and implementation of a low-cost LBL navigation system for an AUV," in OCEANS'99 MTS/IEEE. Riding the Crest into the 21st Century, vol. 2. IEEE, 1999, pp. 774-779.

[6] K. Vickery, "Acoustic positioning systems. a practical overview of current systems," in Autonomous Underwater Vehicles, 1998. AUV'98. Proceedings of the 1998 Workshop on. IEEE, 1998, pp. 5-17.

[7] S.-j. Li, C.-h. Tao, and G.-s. Bao, "INS/USBL underwater navigation system based on Kalman filter," Ocean Technology, vol. 3, no. 14, pp. 47-50, 2008.

[8] X. Yun, E. Bachmann, R. McGhee, R. Whalen, R. Roberts, R. Knapp, A. Healey, and M. Zyda, "Testing and evaluation of an integrated GPS/INS system for small AUV navigation," IEEE Journal of Oceanic Engineering, vol. 24, no. 3, pp. 396-404, 1999.

[9] A. Kim and R. M. Eustice, "Active visual SLAM for robotic area coverage: Theory and experiment," The International Journal of Robotics Research, vol. 34, no. 4-5, pp. 457-475, 2015.

[10] S. Hong, J. Kim, J. Pyo, and S.-C. Yu, "A robust loop-closure method for visual SLAM in unstructured seafloor environments," Autonomous Robots, vol. 40, no. 6, pp. 1095-1109, 2016.

[11] S. Barkby, S. Williams, O. Pizarro, and M. Jakuba, "An efficient approach to bathymetric SLAM," in 2009 IEEE/RSJ International Conference on Intelligent Robots and Systems. IEEE, 2009, pp. 219-224.

[12] E. Chen and J. Guo, "Real time map generation using sidescan sonar scanlines for unmanned underwater vehicles," Ocean Engineering, vol. 91, pp. 252-262, 2014.

[13] D. Ribas, P. Ridao, J. D. Tardós, and J. Neira, "Underwater SLAM in man-made structured environments," Journal of Field Robotics, vol. 25, no. 11-12, pp. 898-921, 2008.
[14] M. Dong, W. Chou, and B. Fang, "Underwater matching correction navigation based on geometric features using sonar point cloud data," Scientific Programming, vol. 2017, pp. 1-10, 2017.

[15] A. Burguera, Y. González, and G. Oliver, "The UspIC: Performing scan matching localization using an imaging sonar," Sensors, vol. 12, no. 6, pp. 7855-7885, 2012.

[16] A. Burguera, "A novel approach to register sonar data for underwater robot localization," in Intelligent Systems Conference (IntelliSys), 2017. IEEE, 2017, pp. 1034-1043.

[17] A. Doucet, N. de Freitas, K. Murphy, and S. Russell, "Rao-Blackwellised particle filtering for dynamic Bayesian networks," in Proceedings of the Sixteenth Conference on Uncertainty in Artificial Intelligence. Morgan Kaufmann Publishers Inc., 2000, pp. 176-183.

[18] G. Grisetti, C. Stachniss, and W. Burgard, "Improved techniques for grid mapping with Rao-Blackwellized particle filters," IEEE transactions on Robotics, vol. 23, no. 1, pp. 34-46, 2007.

[19] R. Smith, M. Self, and P. Cheeseman, "Estimating uncertain spatial relationships in robotics," in Autonomous Robot Vehicles. Springer New York, 1990, pp. 167-193.

[20] M. Montemerlo, N. Roy, S. Thrun, D. Hähnel, C. Stachniss, and J. Glover, "CARMEN-the carnegie mellon robot navigation toolkit," 2002. [Online]. Available: http://carmen.sourceforge.net

[21] S. Thrun, W. Burgard, and D. Fox, Probabilistic Robotics. MIT press Cambridge, 2005, vol. 1.

[22] A. Burguera, Y. Gonzalez, and G. Oliver, "Probabilistic sonar scan matching for robust localization," in 2007 IEEE International Conference on Robotics and Automation. IEEE, 2007, pp. 3154-3160.

[23] “3D simulator-Gazebo," http://www.ros.org/wiki/gazebo, [Online; accessed 28-November-2018].

[24] "Robot Operating System," http://www.ros.org/wiki/, [Online; accessed 28-November-2018].

[25] J. Sturm, N. Engelhard, F. Endres, W. Burgard, and D. Cremers, "A benchmark for the evaluation of RGB-D SLAM systems," in 2012 IEEE/RSJ International Conference on Intelligent Robots and Systems. IEEE, 2012, pp. 573-580. 


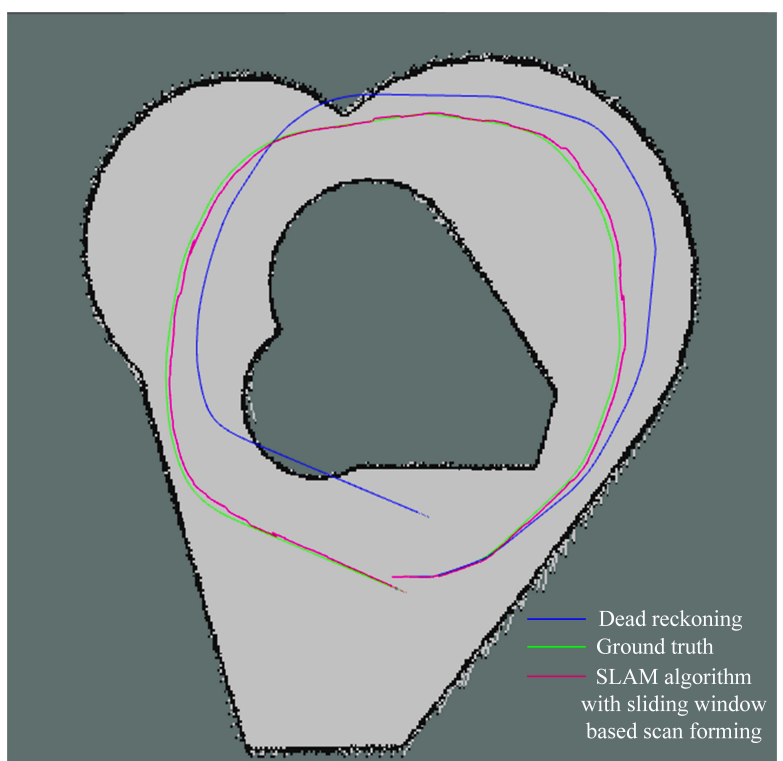

Fig. 10: Occupancy grid map and trajectories produced by SLAM algorithm with sliding window based scan forming incorporating motions.

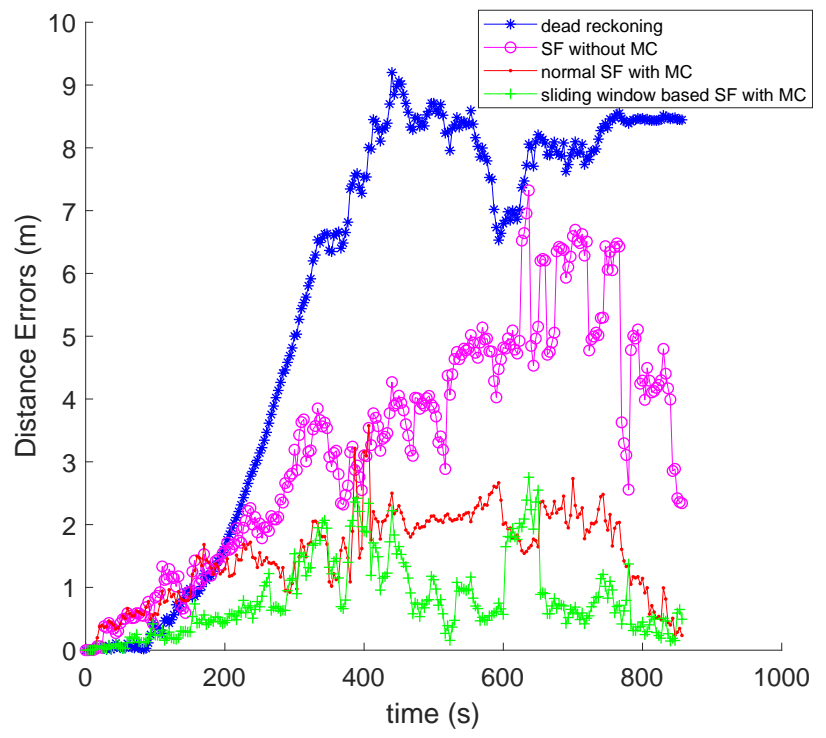

Fig. 11: The relative localization errors between the poses estimated by various algorithms and the ground truth.

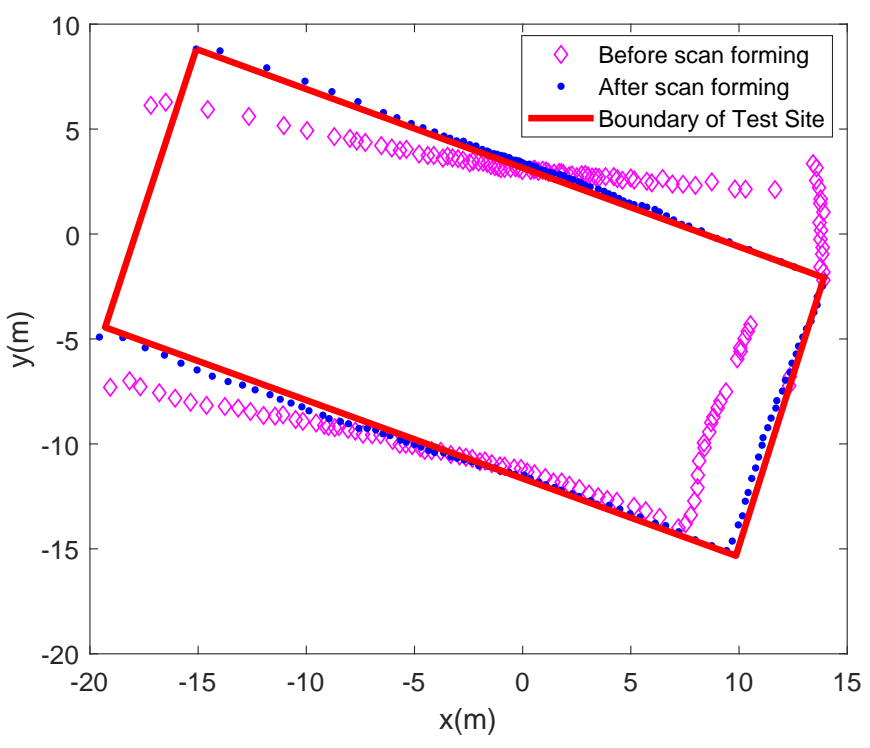

Fig. 13: The effect of scan forming.

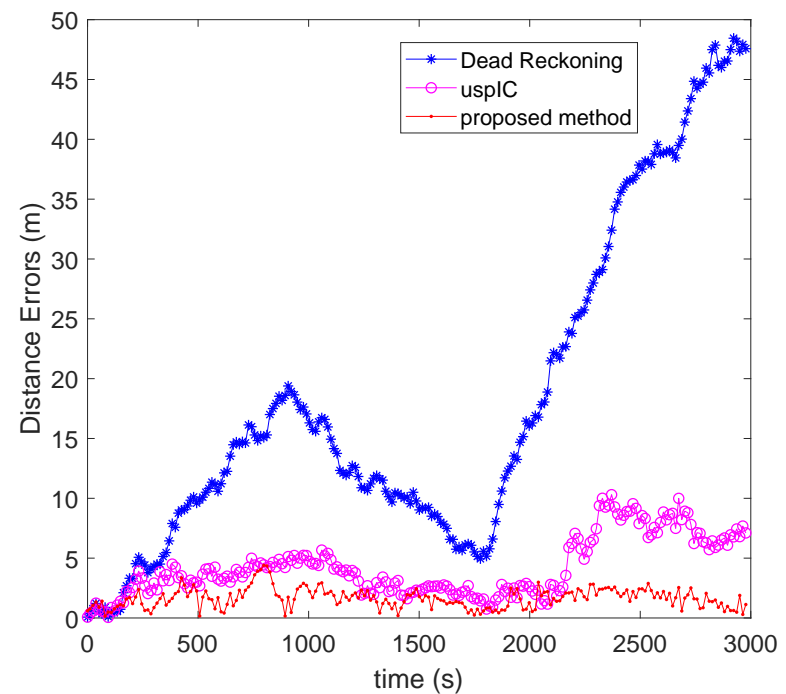

Fig. 17: The relative localization errors among the uspIC trajectories, the proposed SLAM algorithm and the ground truth using data from [13]. 


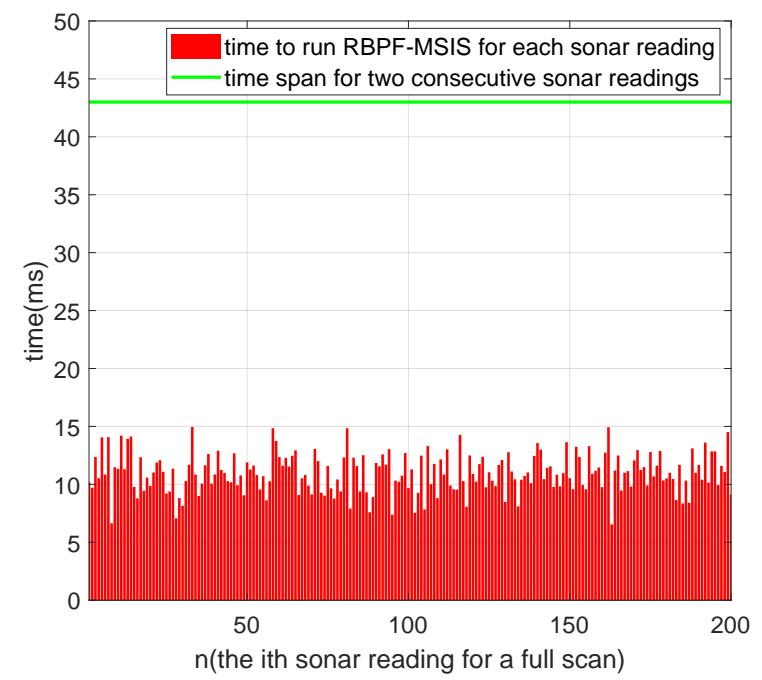

Fig. 18: Time for running RBPF-MSIS after each sonar reading has been received during a full 360-degree scan with SCPBot AUV. 